\title{
Evidence of Human Exposure to Tamdy Virus, Northwest China
}

\author{
Abulimiti Moming, ${ }^{1}$ Shu Shen, ${ }^{1}$ Yaohui Fang, Jingyuan Zhang, Yanfang Zhang, Shuang Tang, \\ Tianxian Li, Zhihong Hu, Hualin Wang, Yujiang Zhang, Surong Sun, Lin-Fa Wang, Fei Deng
}

We report the isolation of Tamdy virus from Hyalomma asiaticum ticks in northwest China and serologic evidence of human Tamdy virus infection in the same region. These findings highlight the need to further investigate a potential causal relationship between Tamdy virus and febrile illnesses of unknown etiology in that region.

$\mathrm{T}$ The species Tamdy orthonairovirus (genus Orthonai1 rovirus, family Nairoviridae) includes 5 viruses: Tamdy virus (TAMV), Burana virus (BURV), Tăchéng tick virus 1 (TcTV-1), Huángpí tick virus 1 (HpTV1 ), and Wēnzhōu tick virus (WzTV) (1). TAMV and BURV were initially isolated from ticks in countries in central Asia (2-4), but little is known about their medical and veterinary importance. TcTV-1, HpTV1 , and WzTV were putative viruses identified by virome sequencing from ticks in China (5); however, their virologic properties and pathogenesis potential remain unclear. One study (6) reported TcTV-1 isolated from a febrile patient in northwest China, providing evidence of the potential public health threat from these viruses. We report TAMV isolated from ticks in northwest China and demonstrate serologic evidence of infection in humans.

\section{The Study}

During April and May of 2016 and 2017, we collected Hyalomma asiaticum ticks ( $\mathrm{n}=4,123)$ from Xinjiang in northwest China and divided the ticks into 55

Author affiliations: Wuhan Institute of Virology, Chinese Academy of Sciences, Wuhan, China (A. Moming, S. Shen, Y. Fang,

J. Zhang, Y. Zhang, S. Tang, T. Li, Z. Hu, H. Wang, F. Deng);

College of Life Science and Technology, Xinjiang University,

Urumqi, China (A. Moming, S. Sun); National Virus Resource

Center, Wuhan (S. Shen, Y. Zhang, S. Tang, F. Deng); Center for

Disease Control and Prevention of Xinjiang Uygur Autonomous

Region, Urumqi, China (Y. Zhang); Programme in Emerging

Infectious Diseases, Duke-NUS Medical School, Singapore

(L-F.. Wang)

DOI: https://doi.org/10.3201/eid2712.203532 groups according to the sampling location $(\mathrm{n}=50-100$ ticks/group) (Figure 1; Appendix Table 1, https:// wwwnc.cdc.gov/EID/article/27/12/20-3532-App1. pdf). We isolated the virus from homogenates of each tick group in suckling mice. After the first inoculation, we observed symptoms in mice including loss of balance, limb paralysis, tremors, and articulo mortis from $4(36.37 \%)$ of 11 pooled samples from Yuli County, 1 (14.29\%) of 7 from the city of Karamay, 3 $(60 \%)$ of 5 from Luntai County, and 17 (53.13\%) of 32 from the city of Wujiaqu (Appendix Table 1). We performed a second inoculation using brain samples from diseased mice from Luntai and Wujiaqu Counties, in which $>50 \%$ of the mice experienced illness onset after first inoculation. Similar symptoms were reproducibly observed in 1 group from Luntai and 4 groups from Wujiaqu (Appendix Table 1).

Subsequently, we prepared 3 RNA pools of diseased mouse brains and obtained a total of $196,946,814$ reads by RNA sequencing. We found TAMV contigs in all 3 pools (Appendix Table 2), confirming findings using real-time reverse transcription PCR (rRT-PCR) (data not shown). We used homogenates of 2 TAMV RNA-positive brain samples from the A-M6 pool (Appendix Table 2) to isolate viruses in Vero E6 cells. As indicated by immunofluorescence assays (IFA) (Appendix), we observed increasing TAMV infection from first to fourth passages in Vero E6 cells, suggesting successful isolation (Appendix Figure 1).

Negative-stain electron microscopy revealed an enveloped spherical viral morphology with a diameter of $\approx 90-110 \mathrm{~nm}$ (Figure 2, panel A). We observed viral particles in cytoplasm and vesicles of infected cells (Figure 2, panel B). Although this screening was not exhaustive, IFAs showing varied susceptibility of different cells lines indicate that TAMV seems to have a broad host range, including humans, monkeys, sheep, dogs, and mice (Appendix Figure 2).

${ }^{1}$ These authors contributed equally to this article. 


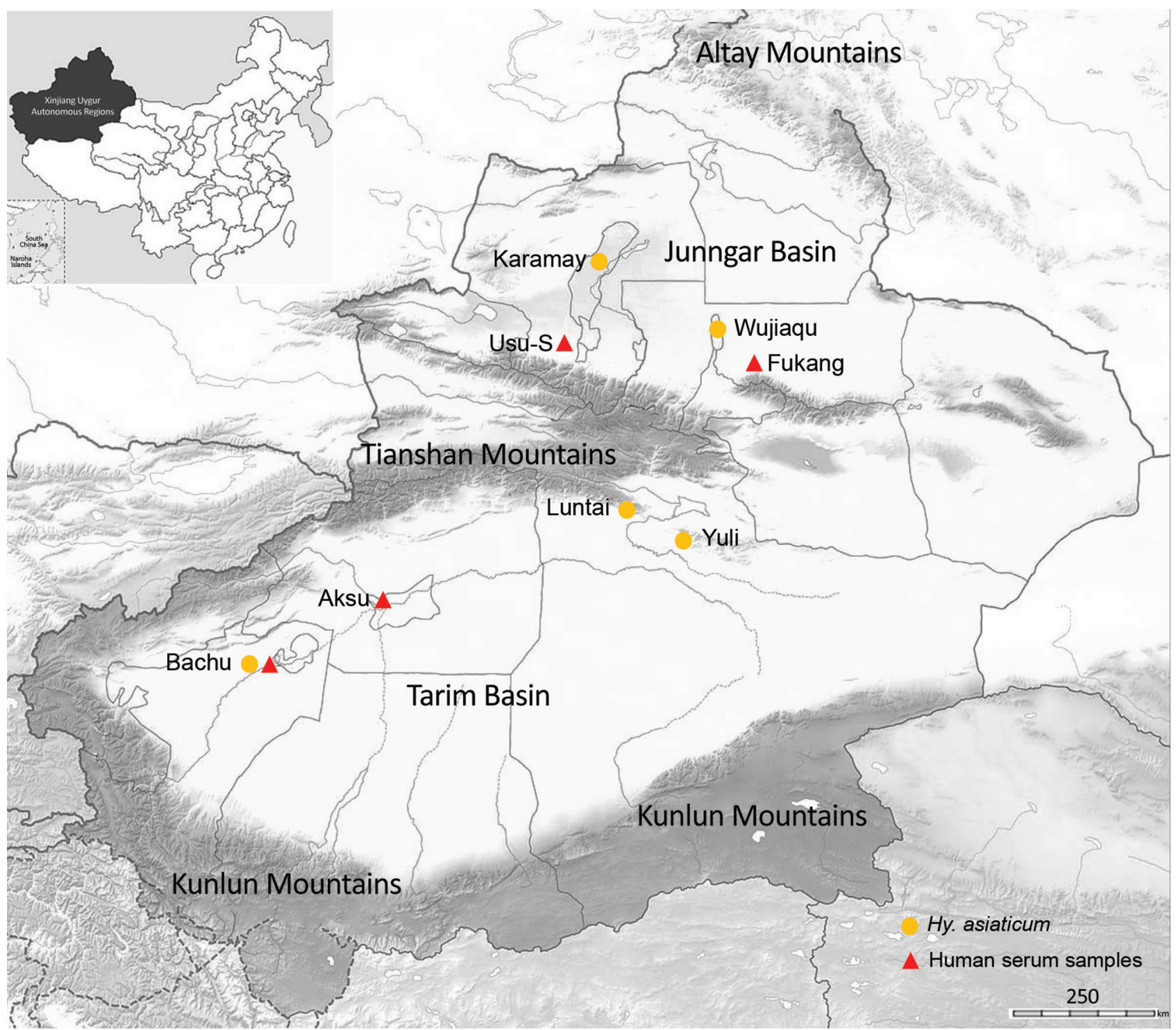

Figure 1. Collection locations for Hyalomma asiaticum ticks and human serum samples used in study of human exposure to Tamdy virus, Xinjiang, China. Usu-S, southern area of Usu City.

The 2 TAMV isolates shared very high sequence similarities $(99.96 \%$ for large, $100 \%$ for medium, and $99.95 \%$ for small segments). We named the 2 TAMV strains YL16082 and YL16083, including an abbreviation (YL) for the geographic location (Yuli County) where the original tick samples were collected. TAMV genome sequences (YL16082) shared $37 \%-59 \%$ identity with other members of the T. orthonairovirus species and $34 \%-49 \%$ identity with Crimean-Congo hemorrhagic fever virus (CCHFV), another Orthonairovirus species. Protein sequences shared $44 \%-62 \%$ identity with other members of the T. orthonairovirus species and $33 \%-40 \%$ with CCHFV (Table 1). Phylogenetic trees based on nucleotide sequences of RNA-dependent RNA polymerase, glycoprotein, and nucleoprotein (NP) genes all confirmed the close taxonomic relationships with currently known TAMV strains and other members of the species T. orthonairovirus (Appendix Figure 3).

To investigate potential human infection by TAMV in northwest China, we conducted a seroprevalence study using archived serum samples from 725 healthy persons (collected in 2005 from Fukang City, 2014 from Aksu City, and 2017 from Usu City) and 87 febrile patients (collected in 2007 from Bachu County, which has a history of CCHFV prevalence) (Appendix). Of the 87 febrile patients, 21 (24.14\%) were TAMV IgG positive and 17 (19.54\%) 

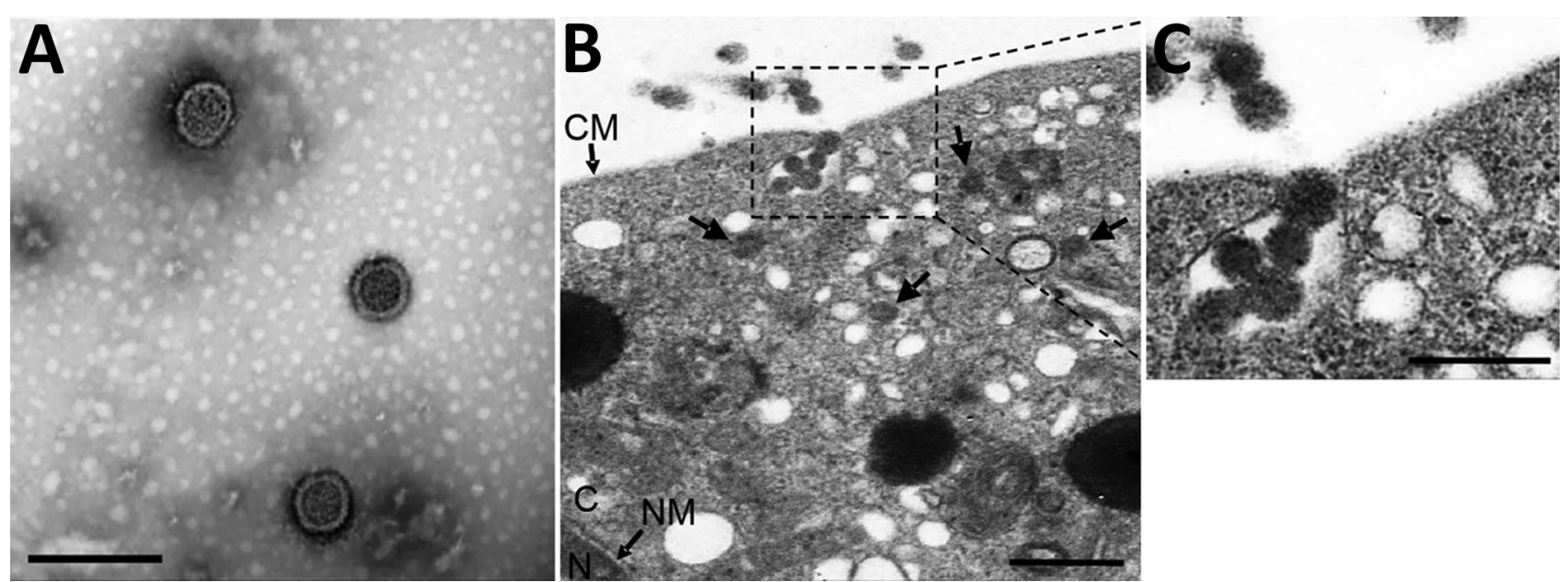

Figure 2. Visualization and subcellular localization of Tamdy virus (TAMV) virions by electron microscopy. A) Negative-staining image of purified TAMV virions. Scale bar indicates $200 \mathrm{~nm}$. B) Image of Vero E6 cells infected with TAMV; arrows indicate TAMV virions in the cytoplasm. Scale bar indicates $500 \mathrm{~nm}$. C) The enlarged image of interest from B. scale bar indicates $200 \mathrm{~nm}$. CM, cell membrane; C, cytoplasm; NM, nuclear membrane; N, nucleus

IgM positive (Table 2; Appendix Table 4), whereas only $1(0.13 \%)$ of the 725 healthy participants we tested IgG positive (data not shown). Neutralization (titers 16-64) was demonstrated in serum samples from 6 febrile patients (6.9\%) (Table 2). Moreover, of the 24 tick groups from the same locations as the febrile patients, 10 groups $(41.76 \%, 6$ identified in sheep and 4 in fields) tested positive for TAMV RNA by rRT-PCR (data not shown). Partial sequences of large segments from these positive groups clustered together with TAMV strains (Appendix Figure 4). These results showed serologic evidence of human exposure to TAMV and evidence of TAMV presence in Hy. asiaticum ticks in northwest China as early as 2007, which warranted more in-depth investigation to establish the potential causal relationship between TAMV and febrile illnesses of unknown etiology in regions where TAMV is present.

Finally, because another T. orthonairovirus, TcTV1 , had been identified in a febrile patient in northwest China (6), we thought it important to determine the potential serologic cross-reactivity between these 2 viruses. However, TAMV and TcTV-1 shared limited protein sequence identity $(49 \%-60 \%)$ (Table
1), suggesting limited cross-reactivity, if any. This result was confirmed by conducting serologic testing using recombinant NP proteins from the 2 viruses. As shown by both IFA and Western blot analyses, TAMV NP antibodies had no cross-reaction with TcTV-1 NP (Appendix Figure 5, panels A, B). In addition, human serum samples that were positive for TAMV IgM or IgG, or both, showed reactivity with TAMV NP, but not with TcTV-1 NP (Appendix Figure 5, panel C).

\section{Conclusions}

TAMV was initially found in ticks in countries in central Asia, including Kazakhstan, Kyrgyzstan, Uzbekistan, and Turkmenistan $(3,4)$. Its infection status in humans and livestock animals was not well characterized. Our data, together with reports of TAMV isolated from ticks in Xinjiang, China (7), and identified in Turkey (8), shows that the geographic distribution of TAMV is much wider than originally recognized. In addition, we provide strong serologic evidence of human exposure in TAMV-affected regions.

Findings of a potential role of TcTV-1 in causing human febrile disease (6) suggest that $>1$ virus in this species group may have the potential to cause

Table 1. Sequence identity of TAMV isolate YL16082 from China compared with other members of the species Tamdy orthonairovirus and Crimean-Congo hemorrhagic fever virus*

\begin{tabular}{|c|c|c|c|c|c|c|}
\hline \multirow[b]{2}{*}{ Virus } & \multicolumn{3}{|c|}{ Nucleotide identity, \% } & \multicolumn{3}{|c|}{ Amino acid identity, $\%$} \\
\hline & L segment & M segment & S segment & RdRp & $\mathrm{G}$ & NP \\
\hline Wēnzhōu tick virus & 59 & 50 & 42 & 62 & 51 & 44 \\
\hline Tăchéng tick virus 1 & 57 & 46 & 44 & 60 & 51 & 49 \\
\hline Huángpí tick virus 1 & 55 & 45 & 42 & 58 & 47 & 46 \\
\hline Burana virus $†$ & 59 & 47 & 37 & 62 & 50 & 44 \\
\hline Crimean-Congo hemorrhagic fever virus & 49 & 34 & 39 & 40 & 33 & 36 \\
\hline
\end{tabular}

${ }^{*} \mathrm{G}$, glycoprotein; L, large; M, medium; NP, nucleoprotein; RdRp, RNA-dependent RNA polymerase; S, small; TAMV, Tamdy virus.

†Partial sequences were available for analyses. 
Table 2 Seroprevalence of TAMV among 87 febrile patients with samples collected in 2007 from Bachu County, Xinjiang, China*

\begin{tabular}{|c|c|c|c|c|c|}
\hline Patient characteristics & Serum samples, no. & $\begin{array}{l}\text { TAMV IgG } \\
\text { positive, } \%\end{array}$ & $\begin{array}{l}\text { TAMV IgM } \\
\text { positive, } \% \\
\end{array}$ & $\begin{array}{c}\text { Neutralization } \\
\text { activity, } \%\end{array}$ & $\begin{array}{c}\text { Neutralization } \\
\text { titers }\end{array}$ \\
\hline \multicolumn{6}{|l|}{ Sex } \\
\hline$M$ & 20 & $3(15.0)$ & $7(35.0)$ & $1(5.0)$ & 64 \\
\hline $\mathrm{F}$ & 29 & $6(20.7)$ & $5(17.2)$ & $3(10.3)$ & $16,32,32$ \\
\hline NA & 38 & $12(31.6)$ & 5 (13.6) & $2(5.3)$ & 16,16 \\
\hline \multicolumn{6}{|l|}{ Age, y† } \\
\hline$<18$ & 6 & $2(33.3)$ & $1(16.7)$ & 0 & \\
\hline $18-30$ & 20 & $1(5.0)$ & $8(40.0)$ & $1(5.0)$ & 16 \\
\hline $31-45$ & 7 & $2(28.6)$ & $1(14.3)$ & 0 & \\
\hline $46-60$ & 11 & $2(18.2)$ & 0 & $1(9.1)$ & 32 \\
\hline$>60$ & 5 & $2(40.0)$ & $2(40.0)$ & $2(40.0)$ & 32,64 \\
\hline Total & 87 & $21(24.1)$ & $17(19.5)$ & $6(6.9)$ & $16-64$ \\
\hline
\end{tabular}

diseases in humans. However, our data did suggest the possibility of such a relationship because the TAMV-positive ratio was much higher among febrile patients than healthy persons in the study from the same region. In addition, at least 2 febrile patients had both TAMV IgM and IgG at the time of sampling, during or not long after acute illness; 1 of them had neutralization to TAMV (Appendix Table 3).

Among study limitations, the nature of using archived samples limited our ability to provide direct evidence of a causal relationship between TAMV and human febrile illnesses. Also, it is possible that the high TAMV antibody-positive ratio might have resulted not from the recent cases but from a small outbreak of human TAMV infection in northwest China in 2007.

In summary, our study strongly suggests the potential of TAMV as a human pathogen and supports an urgent need to conduct more in-depth epidemiologic and pathogenesis investigations into this group of viruses in China, central Asia, and beyond. While the world's attention is currently on coronavirus disease and batborne viruses, our study highlights the need to pay attention at the same time to emerging zoonoses of tick origin to prevent future outbreaks.

\section{Acknowledgments}

We thank the core facility and technical support groups of Wuhan Institute of Virology for providing technical support for electron microscopy analysis, animal experiments, and experimental activities in a Biosafety Level 3 laboratory.

This work was mainly supported by the Science and Technology Basic Work Program (2013FY113500) from the Ministry of Science and Technology of China, the Intergovernmental Special Program of State Key Research and Development Plan from the Ministry of Science and Technology of China (2016YFE0113500), the National
R\&D Infrastructure and Facility Development Program of China, "Fundamental Science Data Sharing Platform" (Y706061YZ1), Science Foundation of China (81690369, 81760365), and the Science Research Key Project of Xinjiang Education Department (XJEDU2019I002). Work in L.-F.W.'s group is supported in part by NRF grants NRF2016NRF-NSFC002-013 and NRF2018NRFNSFC003SB-002, CD-PHRG grant CDPHRG/0006/ 2014, NMRC grant ZRRF16006, and MINDEF grant DIRP2015-9016102060.

\section{About the Author}

Mr. Moming is a doctoral student in a joint training project at the College of Life Science and Technology, Xinjiang University, and Wuhan Institute of Virology, Chinese Academy of Sciences. His main research interests include epidemiologic investigation, identification, and characterization of tick-borne viruses.

\section{References}

1. Abudurexiti A, Adkins S, Alioto D, Alkhovsky SV, Avšič-Županc T, Ballinger MJ, et al. Taxonomy of the order Bunyavirales: update 2019. Arch Virol. 2019;164:1949-65. https:/ / doi.org/10.1007/s00705-019-04253-6

2. L'vov DK, Al'khovskiı SV, Shchelkanov MI, Shchetinin AM, Deriabin PG, Gitel'man AK, et al. Taxonomic status of the Burana virus (BURV) (Bunyaviridae, Nairovirus, Tamdy group) isolated from the ticks Haemaphysalis punctata Canestrini et Fanzago, 1877 and Haem. concinna Koch, 1844 (Ixodidae, Haemaphysalinae) in Kyrgyzstan [in Russian]. Vopr Virusol. 2014;59:10-5.

3. L'vov DK, Sidorova GA, Gromashevskiǔ VL, Skvortsova TM, Aristova VA. Isolation of Tamdy virus (Bunyaviridae) pathogenic for man from natural sources in Central Asia, Kazakhstan and Transcaucasia [in Russian]. Vopr Virusol. 1984;29:487-90.

4. Lvov DK, Sidorova GA, Gromashevsky VL, Kurbanov M, Skvoztsova LM, Gofman YP, et al. Virus "Tamdy" - a new arbovirus, isolated in the Uzbee S.S.R. and Turkmen S.S.R. from ticks Hyalomma asiaticum asiaticum Schulee et Schlottke, 1929, and Hyalomma plumbeum plumbeum Panzer, 1796. Arch Virol. 1976;51:15-21. https:/ / doi.org/10.1007/BF01317830

5. Li CX, Shi M, Tian JH, Lin XD, Kang YJ, Chen LJ, et al. 
Unprecedented genomic diversity of RNA viruses in arthropods reveals the ancestry of negative-sense RNA viruses. eLife. 2015;4:e05378. https:/ / doi.org/10.7554/ eLife. 05378

6. Liu X, Zhang X, Wang Z, Dong Z, Xie S, Jiang M, et al. A tentative Tamdy orthonairovirus related to febrile illness in northwestern China. Clin Infect Dis. 2020;70:2155-60. https:/ / doi.org/10.1093/cid/ciz602

7. Zhou H, Ma Z, Hu T, Bi Y, Mamuti A, Yu R, et al. Tamdy virus in Ixodid ticks infesting bactrian camels, Xinjiang, China, 2018. Emerg Infect Dis. 2019;25:2136-8. https://doi.org/10.3201/eid2511.190512

8. Brinkmann A, Dinçer E, Polat C, Hekimoğlu O,
Hacioğlu S, Földes K, et al. A metagenomic survey identifies Tamdy orthonairovirus as well as divergent phlebo-, rhabdo-, chu- and flavi-like viruses in Anatolia, Turkey. Ticks Tick Borne Dis. 2018;9:1173-83. https://doi.org/10.1016/j.ttbdis.2018.04.017

Address for correspondence: Linfa Wang, Programme in Emerging Infectious Diseases, Duke-NUS Medical School, 169857, Singapore; email: linfa.wang@duke-nus.edu.sg; Fei Deng, Wuhan Institute of Virology, Chinese Academy of Sciences, Xiaohongshan 44, Wuchang District, Wuhan City, Hubei Province, 430071, China; email: df@wh.iov.cn

\section{High-Consequence Pathogens}

- Blastomycosis Surveillance in 5 States, United States, 1987-2018

- Reemergence of Human Monkeypox and Declining Population Immunity in the Context of Urbanization, Nigeria, 2017-2020

- Animal Reservoirs and Hosts for Emerging Alphacoronavirsuses and Betacoronaviruses

- Difficulties in Differentiating Coronaviruses from Subcellular Structures in Human Tissues by Electron Microscopy

- Characteristics of SARS-CoV-2 Transmission among Meat Processing Workers in Nebraska, USA, and Effectiveness of Risk Mitigation Measures

- Systematic Review of Reported HIV Outbreaks, Pakistan, 2000-2019

- Infections with Tickborne Pathogens after Tick Bite, Austria, 2015-2018

- Emergence of Burkholderia pseudomallei Sequence Type 562, Northern Australia

- Histopathological Characterization of Cases of Spontaneous Fatal Feline Severe Fever with Thrombocytopenia Syndrome, Japan

- COVID-19-Associated Pulmonary Aspergillosis, March-August 2020

- Rare Norovirus GIV Foodborne Outbreak, Wisconsin, USA

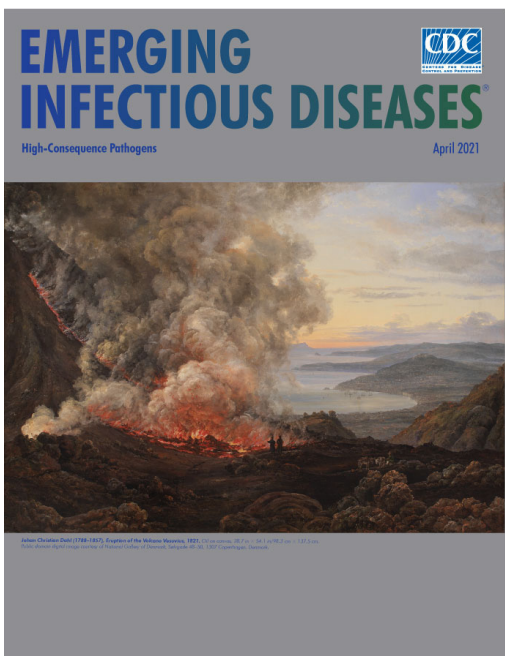

- Genomic Surveillance of a Globally Circulating Distinct Group W Clonal Complex 11 Meningococcal Variant, New Zealand, 2013-2018

- Dynamic Public Perceptions of the Coronavirus Disease Crisis, the Netherlands, 2020

- Evolution of Sequence Type 4821 Clonal Complex Hyperinvasive and Quinolone-Resistant Meningococci

- Epidemiologic and Genomic Reidentification of Yaws, Liberia

- Increased SARS-Cov-2

Testing Capacity with Pooled Saliva Samples
- Sexual Contact as Risk Factor for Campylobacter Infection

- Venezuelan Equine Encephalitis Complex Alphavirus in Bats, French Guiana

- Stability of SARS-CoV-2 RNA in Nonsupplemented Saliva

- Experimental SARS-CoV-2 Infection of Bank Voles

- Analysis of Asymptomatic and Presymptomatic Transmission in SARS-CoV-2 Outbreak, Germany, 2020

- Characteristics and Risk Factors of Hospitalized and Nonhospitalized COVID-19 Patients, Atlanta, Georgia, USA, March-April 2020

- Improving Treatment and Outcomes for Melioidosis in Children, Northern Cambodia, 2009-2018

- Eastern Equine Encephalitis Virus in Mexican Wolf Pups at Zoo, Michigan, USA

- Genomic Analysis of Novel Poxvirus Brazilian Porcupinepox Virus, Brazil, 2019

- Highly Pathogenic Avian Influenza Clade 2.3.4.4 Subtype H5N6 Viruses Isolated from Wild Whooper Swans, Mongolia, 2020

- SARS-CoV-2 Seropositivity among US Marine Recruits Attending Basic Training, United States, Spring-Fall 2020

\section{EMERGING INFECTIOUS DISEASES}

